\title{
Space Weathering Experiments on Spacecraft Materials
}

\author{
D. P. Engelhart ${ }^{1}$, R. Cooper ${ }^{2}$, H. Cowardin ${ }^{3}$, J. Maxwell ${ }^{4}$, E. Plis ${ }^{5}$, D. Ferguson ${ }^{2}$, D. Barton², S. Schiefer ${ }^{2}$, R. \\ Hoffmann ${ }^{2}$
}

1. National Research Council at Air Force Research Laboratory, Albuquerque, NM, 87117, USA

2. Air Force Research Laboratory, Space Vehicles Directorate, Kirtland AFB, Albuquerque, NM, 87117, USA

3. University of Texas-El Paso-Jacobs JETS Contract, NASA Johnson Space Center, Houston, TX 77058

4. University of Colorado-Boulder, Department of Aerospace Engineering, Boulder, CO

5. Assurance Technology Corp., 84 South Street, Carlisle, MA 01741

A project to investigate space environment effects on specific materials with interest to remote sensing was initiated in 2016. The goal of the project is to better characterize changes in the optical properties of polymers found in multilayered spacecraft insulation (MLI) induced by electron bombardment. Previous analysis shows that chemical bonds break and potentially reform when exposed to high energy electrons like those seen in orbit. These chemical changes have been shown to alter a material's optical reflectance, among other material properties. This paper presents the initial experimental results of MLI materials exposed to various fluences of high energy electrons, designed to simulate a portion of the geosynchronous Earth orbit (GEO) space environment. It is shown that the spectral reflectance of some of the tested materials changes as a function of electron dose. These results provide an experimental benchmark for analysis of aging effects on satellite systems which can be used to improve remote sensing and space situational awareness. They also provide preliminary analysis on those materials that are most likely to comprise the high areato-mass ratio (HAMR) population of space debris in the geosynchronous orbit environment. Finally, the results presented in this paper serve as a proof of concept for simulated environmental aging of spacecraft polymers that should lead to more experiments using a larger subset of spacecraft materials.

\section{INTRODUCTION}

Over the last decade, astronomical reflectance spectroscopy has been proposed to characterize the material composition of artificial space objects, such as satellites, rocket bodies, and human-made debris.[1, 2] In this application, reflected light is collected from a remote target illuminated by a continuous source (the Sun). The reflected light produces a spectrum whose shape and absorption features are indicative of a specific material composition. Since each material has a unique spectral fingerprint, it may be unambiguously identified if its spectral features are well differentiated.

In general, it has been found that the space environment fundamentally changes spacecraft materials.[3-5] The nature of particles primarily responsible for damage is dependent on the orbit; in geosynchronous Earth orbit (GEO), high energy electrons are the primary damaging species in terms of energy deposition. [6, 7] Solar energetic protons are also a major component of the GEO environment but have a lower average flux which tends to be transient, corresponding to the solar cycle. Future studies will include damage from both electrons and protons. There have been many studies reporting modification of polymer properties under electron irradiation.[8-10] However, these studies have been mainly descriptive in nature with no real attempts to understand the radiation-induced chemical modifications that drive the changes in material optical properties.

Recently, we undertook an effort to understand the chemical changes that occur in polyimide (PI, Kapton- ${ }^{\circledR}$ ), when it is exposed to high energy electrons.[11, 12] It was found that new chemical structures originating from a radiationinduced breakage of phenyl rings and/or ether bridges are formed in the radiation-damaged PI. These new chemical structures give rise to new lower energy electronic states than are present in the pristine material as demonstrated by a red-shift in the optical absorption edge of the material. This dramatic change in the material's optical properties suggests that a careful study of the effects of space weather aging on commonly used spacecraft materials could enable identification and characterization of satellites and debris on orbit via ground- and space-based optical observations.

In this paper, we present an investigation of the changes in optical reflectance of constituents of multi-layered insulation during exposure to a simulated GEO environment. MLI is a material commonly used on rocket bodies and 
satellites which uses multiple radiation-heat transfer barriers to tune energy transfer at a spacecraft surface.[13] Moreover, a space debris population recently was found with high area-to-mass ratio which could be MLI.[14, 15] Development of a predictive model for the optical properties of MLI as a function of exposure to the space environment will aid telescopic identification of known artificial space objects and be the first step toward characterization of unknown objects.

MLI can be comprised of a variety of materials; typically, the outer layers are outward facing amber-colored PI layers with inward facing aluminized backing. The inner layers consist of alternating DACRON or Nomex netting and aluminized Mylar as seen in Fig. 1. We assessed the reflectivity of different MLI constituents (excluding the Nomex netting) in situ during irradiation with high-energy $(90 \mathrm{keV})$ electrons. Next, radiation dose-dependent color- color index data were prepared for those samples that exhibited optical changes after electron irradiation. An r' -i' color index is presented, using Sloan Digital Sky Survey (SDSS) filters.[16] Color index data can assist in determination of remote target material composition. [i] Due to the instrumentation used for this preliminary study, the wavelength regime investigated spans (450-1000 $\mathrm{nm}$ ), therefore the SDSS filters were used for color-color investigations rather than the Johnson/Bessell system. Color-Color spectral indexing is useful because a shift in a space object's color index could be used for identification and characterization.

\section{SAMPLES INVESTIGATED}

The space-facing side of MLI consists of a PI sheet with a vapor deposited aluminum backing. Previous work has indicated that high energy electrons alter the chemical structure of PI, leading to changes in the optical transmission spectrum. When illuminated from the PI side, the aluminum backing on the space-facing MLI layer acts as a mirror, reflecting a significant portion of the light transmitted through PI. In this way, a reflection measurement is actually a superposition of a reflection spectrum and a double-pass transmission/absorption spectrum, generating an optical fingerprint which exhibits similar characteristics to the pure transmission spectrum of polyimide. The sample of MLI studied here consisted of 4 distinct layers with Nomex netting interposed between each, as shown in Figure 1.

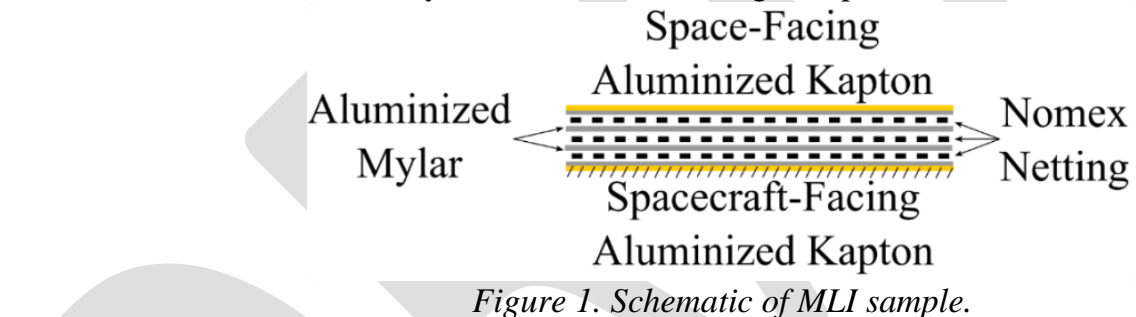

Investigated samples were prepared by separating the layers of an MLI sample (81205; ASSY290-21098-1;L/N 93073; 11-2-92). In total, five different MLI constituents were identified, as shown in Table 1 along with the total thickness of each. The aluminized spacecraft-facing Kapton layer is perforated to allow materials to degas efficiently after entering orbit. The Nomex netting was sparse enough that no reliable signal could be measured through spectroscopy. This material was therefore discarded from the study. There is a structural distinction between the space facing aluminized PI and the spacecraft facing PI. The spacecraft facing layer has a reinforcing netting integrated into the film that can be seen in sample position 5 and 6 in Figure 2. It is not known what this netting is composed of, but we assume Kevlar or something similar.

Samples were placed on a rotating aluminum carousel, as shown in Figure 2 using small tabs of PI adhesive tape. Acktar light absorbent foil, a highly absorptive, optically diffuse foil, was placed behind each sample to minimize the optical signal from the carousel. One piece of Acktar light absorbent foil was also aged so that its response to the GEO environment could be characterized for data reduction purposes. Both sides of the space-facing and spacecraft-facing aluminized Kapton were aged and characterized. The two sides of aluminized Mylar are identical, so only one side of each layer was aged. Details of the samples mounted on the aluminum carousel are outlined in Table 1. Before mounting, all samples were subject to a $24 \mathrm{hr}$ vacuum bake at $60^{\circ} \mathrm{C}$ and allowed to degas in the experimental chamber, discussed in Section 2, at $<10^{-6}$ Torr overnight before aging was begun. 


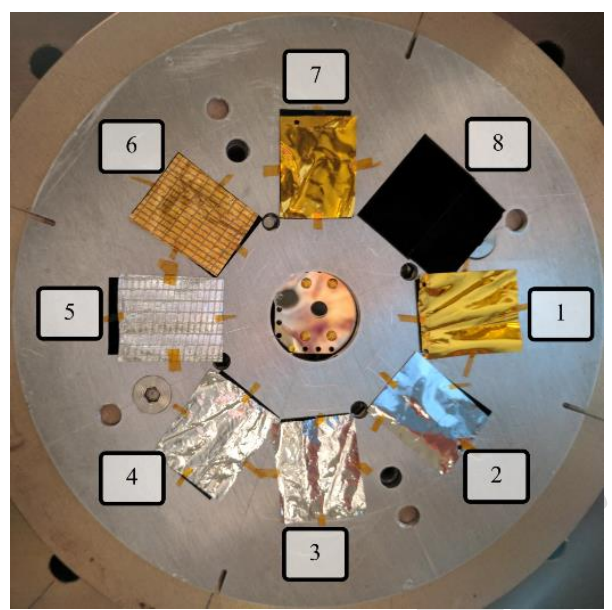

Figure 2 Samples mounted on aluminum carousel. Material identifications are listed in Table 1

Table 1. Material composition, thickness, and measured side

\begin{tabular}{|c|c|}
\hline Sample Position & Material (side, thickness) \\
\hline 1 & Space-Facing Alumnized Kapton (Kapton Side, 114 $\mu \mathrm{m})$ \\
\hline 2 & Space-Facing Alumnized Kapton (Aluminum Side, 114 $\mu \mathrm{m})$
\end{tabular}

The electrical properties of Acktar light absorbent foil were measured in order to verify that it was safe to use in the electron beam and wouldn't arc or contaminate the other sample. The dark resistivity and bulk conductivity were evaluated with the surface potential decay (SPD) method [12] and were found to be $3.8 \times 10^{19} \Omega \cdot \mathrm{cm}$ and $2.6 \times 10^{-20}$ $(\Omega \cdot \mathrm{cm})^{-1}$, respectively. The initial (maximum) voltage, $\mathrm{V}_{\mathrm{o}}$ accumulated by the material immediately after electron bombardment was $-62 \mathrm{~V}$. Thus, although the conductivity of the Acktar foil is low, it does not charge significantly under electron bombardment and the possibility of arcing between samples and grounded aluminum plate is minimal.[17]

\section{JUMBO ENVIRONMENTAL CHAMBER}

Space weather in GEO is a combination of high vacuum, electron $(0.01-10 \mathrm{MeV})$ and proton $(10-4-10 \mathrm{MeV})$ fluxes, solar radiation, and thermocycling between 120-420 K.[18] In terms of total dose experienced by an object in GEO over several solar cycles, electrons are the dominant source of energy deposition. For this reason we have chosen to focus this study on electron damage only. However, the literature suggests that solar protons while lower in total flux and wildly variable in energy may also play a significant role in the evolution of spacecraft materials. This work is critical to understanding the life cycle of spacecraft but, will be left for future studies. [19-21] 
To simulate an electron dose typical to the GEO environment, samples were bombarded with high energy (90 keV) mono-energetic electron radiation from a Kimball Physics EG8105-UD electron flood gun in the Spacecraft Charging and Instrument Calibration Laboratory (SCICL) at Kirtland Air Force Base in New Mexico, USA.[22]

As samples are bombarded with high energy electrons, the carousel is rotated so that hot spots in the beam do not cause substantially different doses between different samples. A Faraday cup can be seen in the center of the carousel in Figure 2 allowing us to continuously measure the electron flux and accurately calculate the total dose.

Radiation dose received by each sample was calculated using the spatial map of the electron aging beam, the simulated dose profile, and the duration of material irradiation. Monte-Carlo simulations using Monte Carlo N-Particle Code, v. 6.1 (MCNP6.1)[23] were performed in order to accurately characterize the electron dose profile. In this simulation, 20 million $90 \mathrm{keV}$ electrons impinged on front and back sides of $171 \mu \mathrm{m}$ thick aluminized spacecraft facing Kapton, $114 \mu \mathrm{m}$ thick aluminized space facing Kapton, and $15.5 \mu \mathrm{m}$ thick aluminized Mylar. The thickness of last sample was chosen as an average between $14 \mu \mathrm{m}$ and $17 \mu \mathrm{m}$ thick aluminized Mylar pieces constituting the MLI. The simulation results presented in Figure 3 show the average energy deposition per electron per $1 \mu \mathrm{m}$ thick sample layer. Backscattering and bremsstrahlung were also accounted for in the simulation.

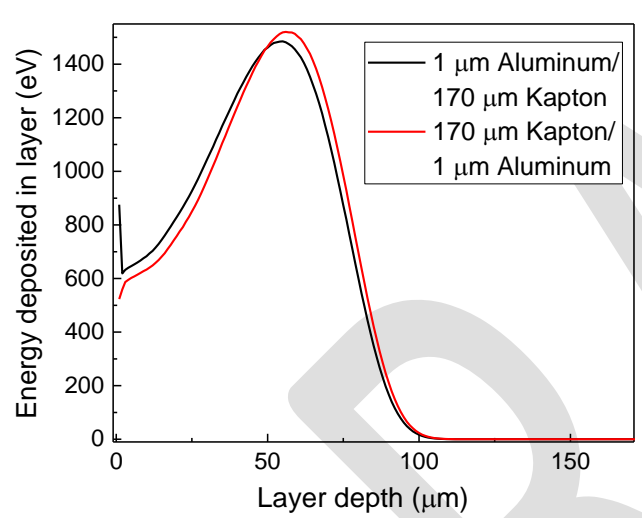

(a)

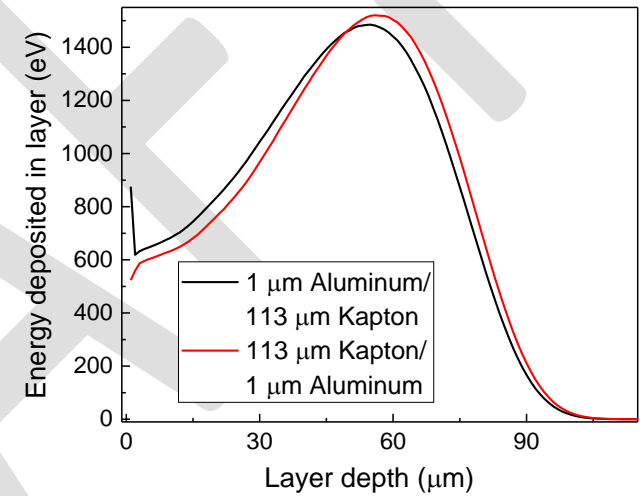

(b)

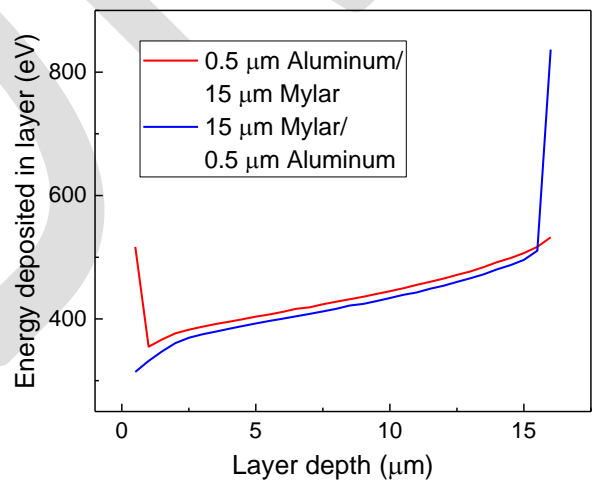

(c)

Figure 3. Simulated energy deposition in studied samples by $90 \mathrm{keV}$ electron beam. (a) front and back of $171 \mu \mathrm{m}$ thick aluminized spacecraft facing Kapton, (b) front and back of $114 \mu \mathrm{m}$ thick aluminized space facing Kapton, (c) front and back of $15 \mu \mathrm{m}$ thick aluminized Mylar with thick backing aluminium layer.

The Monte-Carlo simulations predict a maximum penetration depth of $\sim 100 \mu \mathrm{m}$, slightly deeper than that predicted by the CSDA approximation $(91 \mu \mathrm{m})$. In order to approximate the "GEO age," i.e. the approximate time on orbit a 
piece of similar material would have to spend in GEO orbit to experience this amount of electron induced energy deposition (and, presumably, damage), we converted the energy deposited by the $40 \mathrm{nA} / \mathrm{cm} 2$ electron beam into Gy (joule $/ \mathrm{kg}$ ) and the corresponding age was calculated using the AE9 space plasma model, which predicts an energetic dose rate of $0.215 \mathrm{~Gy} / \mathrm{s}$ in GEO orbit.[6] Using these approximations, the maximum dose for each material was calculated to be equivalent to 37.6 MGy (5.5 GEO years) for $117 \mu \mathrm{m}$ Kapton, 25.1 MGy (3.7 GEO years) for $171 \mu \mathrm{m}$ Kapton, and 12.5 MGy (1.6 GEO years) $15.5 \mu$ m Mylar.

\section{OPTICAL DATA ACQUISITION}

Periodically throughout the aging process, reflectance spectra were recorded using an in-vacuum integrating sphere so that the measured quantity is directional hemispherical reflectance (DHR). Because it was not possible to measure the DHR with the integrating sphere without blocking the electron beam, a series of automated in-vacuum motions was required. For each measurement, an ASD FieldSpec Pro spectroradiometer operating in $350 \mathrm{~nm}-2500 \mathrm{~nm}$ range collects first a white reference spectrum from a piece of in-vacuum Spectralon manufactured and calibrated by LabSphere. This highly reflective, diffuse surface, along with the provided calibration curve, allows the signal collected of a given sample to be converted from raw counts to absolute reflectance. The electron beam is momentarily extinguished and the motion system moves the integrating sphere over to the sample carousel where it measures samples as they are rotated into the measurement position. Dark current images were taken for every set of measurements in order to subtract electronic noise from the optical measurements.

Figure 4 shows a block diagram of the experiment. The spectroradiometer and halogen light source are placed outside the chamber, while the samples, integrating sphere and Spectralon are within. This necessitates four fiber optic cables, two in vacuum and two out of vacuum, as well as two fiber optic vacuum feedthroughs. The six elements used all exhibit high attenuation above $2100 \mathrm{~nm}$. While the optical elements described above have relatively high throughput at wavelengths below $2100 \mathrm{~nm}$, light is lost at each interface and in each element. The chosen source, HL-2000-HAS from Ocean Optics, produces high intensity light between $400 \mathrm{~nm}$ and $2400 \mathrm{~nm}$. The combination of this source and the optical elements described above results in a measurement range with sufficient signal to noise ratio between 450 $\mathrm{nm}$ and $1800 \mathrm{~nm}$. For those materials in which changes were detected, the optical signatures changed between 450$1000 \mathrm{~nm}$.

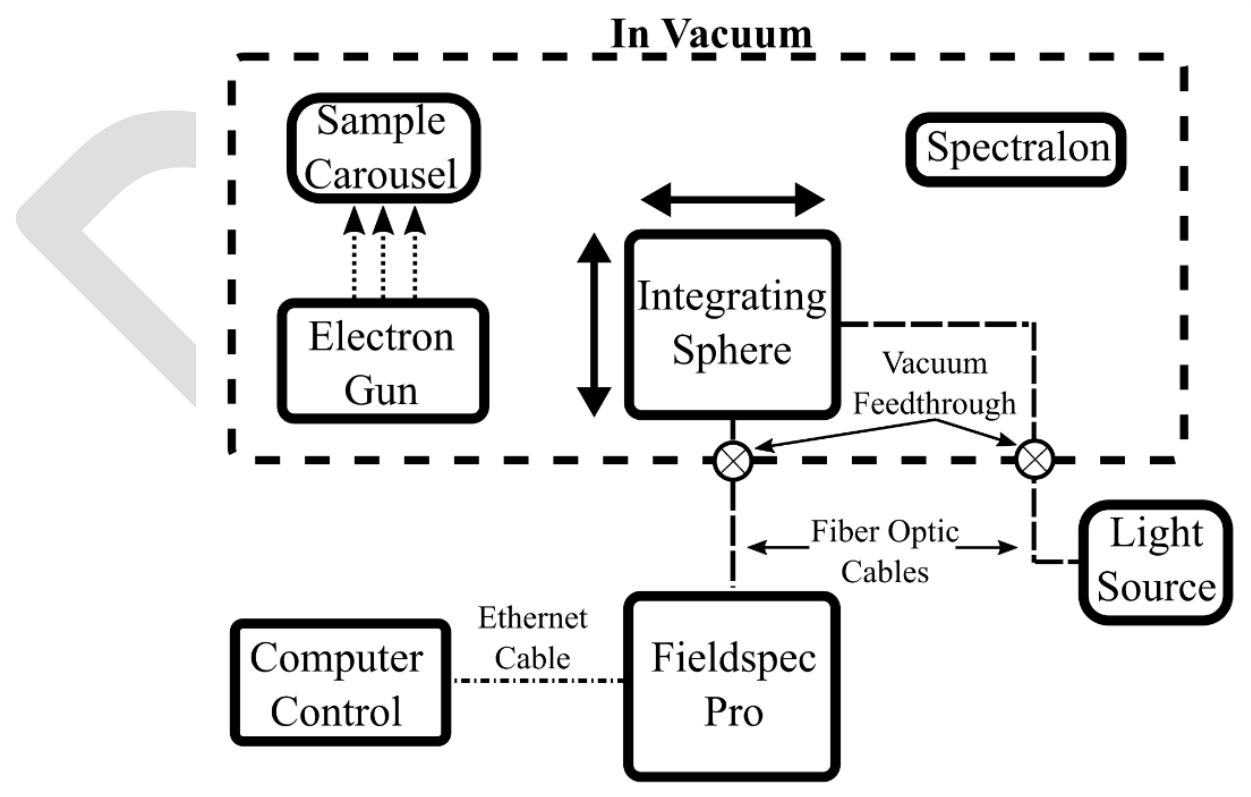

Figure 4. Block diagram of experiment.

To avoid collecting stray light, the integrating sphere is mounted on a spring-loaded ball joint which aligns outer surface of the integrating sphere assembly to the measured object when pushed up against it by the 3D motion stage within the vacuum chamber. 


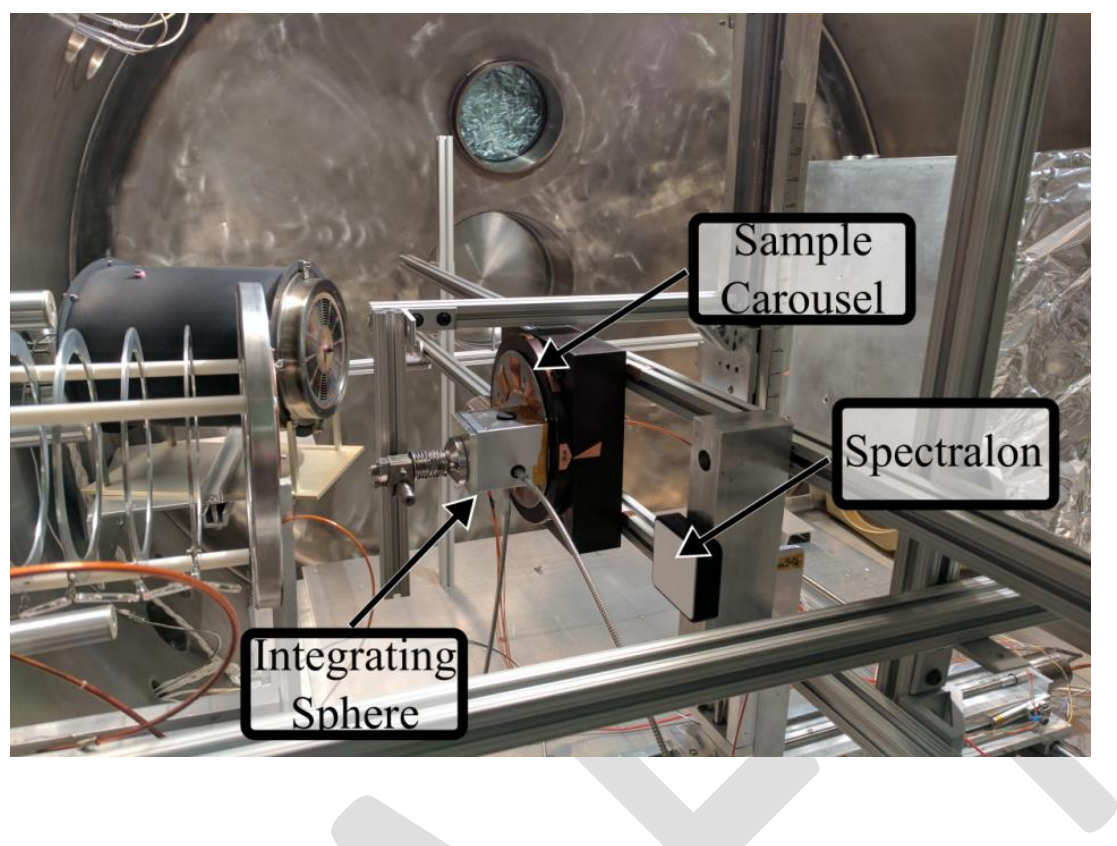

Figure 5. Experimental setup in the vacuum chamber, electron flood gun not pictured

The integrating sphere, which is housed in an aluminum chassis, covers the Spectralon whenever the gun is emitting high energy electrons. This ensures that the Spectralon, which is made of a dielectric material, does not charge or experience damage from high energy electrons that could cause its reflectance to deviate from the calibration. A retarding grid which cuts off the electron beam is engaged whenever measurements take place.

\section{RESULTS}

The transformation from raw counts from the spectrometer to the absolute reflectance is shown in below in Eq. 1:

Eq. 1

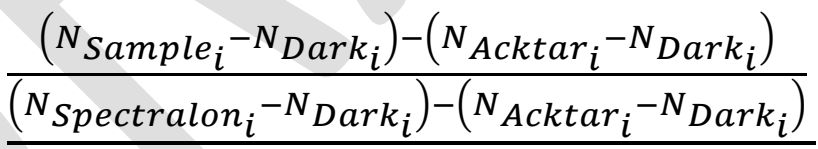

$$
\begin{aligned}
& \text { Reflectance }_{\text {Spectralon }} \text { Calibrated }
\end{aligned}
$$

The iterator i indicates the measurement step at which the values were taken. Each measurement step follows the procedure

1. Take Dark Spectrum

2. Take Spectralon Spectrum

3. Take Samples 1 through 8 Spectra

The subtraction of Acktar Spectral Black serves to remove the light that is scattered from the IS without interacting with the sample. Figure 1 shows the spectrum of this material as published on Acktar's website.[24] The material works well as a black standard between 400-2500nm. We assume here that Acktar has a 0\% reflectance that we know is only approximately true and leads to additional uncertainty in the calculated reflectance. By taking spectra of the Acktar, the system's drift and light scattered directly from the integrating sphere to the spectrometer can be factored out of the material's reflectance measurements. Finally, division by the calibrated reflectance spectrum of the Spectralon panel allows for the conversion from relative reflectance to absolute reflectance.

The absolute reflectance for each material was measured as the material was aged in the electron beam. For each of the materials the absolute reflectance was plotted as a function of dose in Figure 6. 


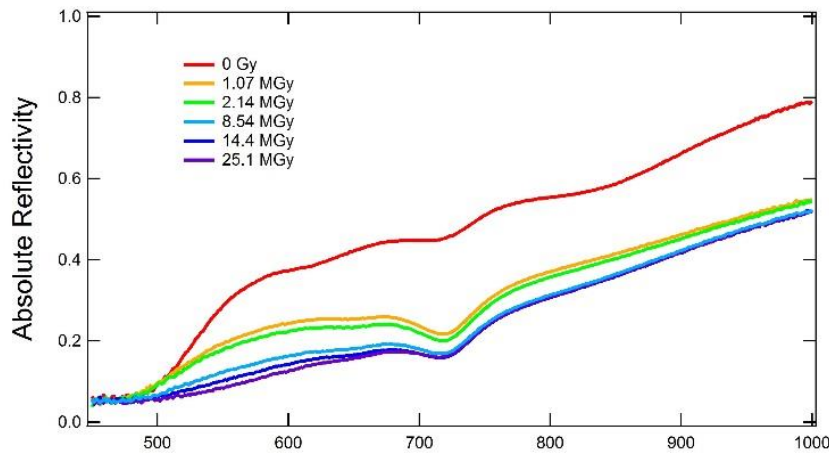

a)

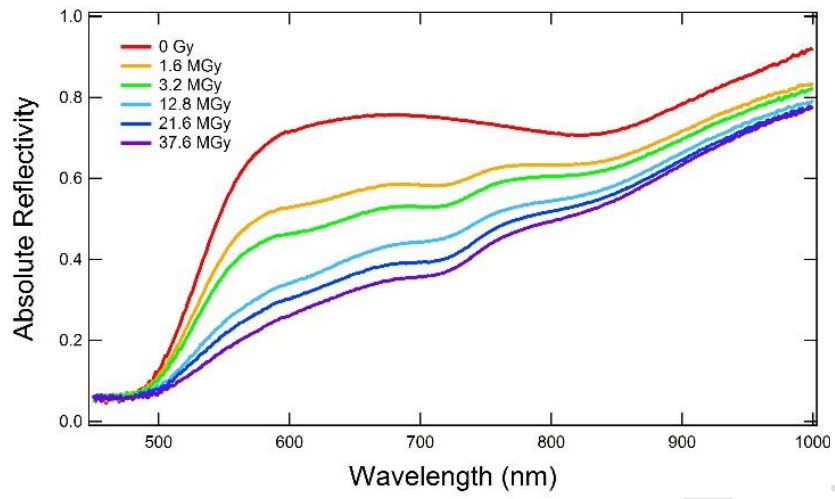

c)

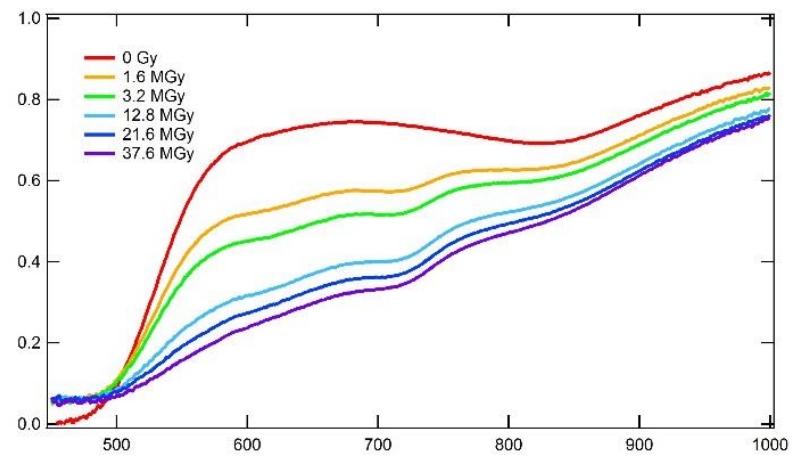

b)

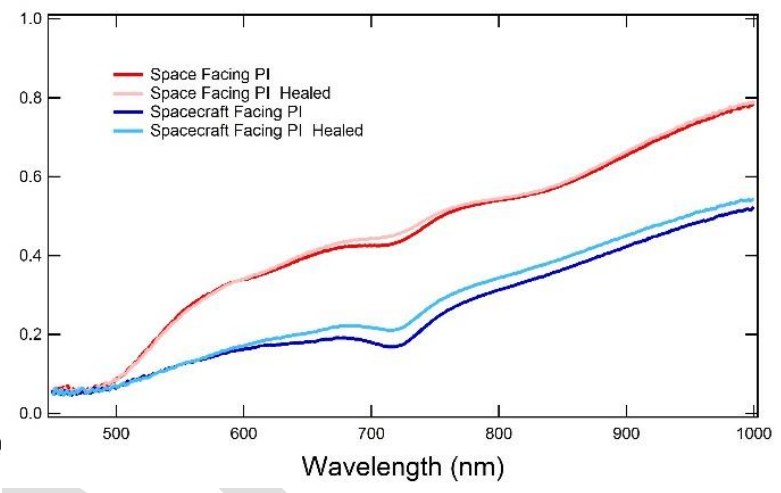

d)

Figure 6. Reflectance spectra of various materials from table 2 at several electron doses. a) spacecraft facing PI (material 6), b) Space facing PI (material 1), and c) Space facing PI (material 7). d) Spectrum of space facing and spacecraft facing PI before and after $65 \mathrm{hr}$ of healing in vacuum.

The aluminum sputtered Mylar did not exhibit a change in absolute reflectance after exposure to electron radiation. This is physically reasonable as no chemical effect can be expected from electron bombardment of the metal. Laboratory observations, however, indicated embrittlement of the material, indicating that the Mylar sandwiched between the aluminum layers had been degraded by the electron beam. The Kapton components exhibited extensive optical changes after radiation in the visible and near IR spectra, however. These changes can be seen in Figure 6. New experiments are planned to characterize the spectral signature of the materials out to $2500 \mathrm{~nm}$. Although material 6 (panel a in Figure 6) is primarily composed of aluminized Kapton, similar to materials 1 and 7 (panels b and $\mathrm{c}$ in same figure), there is a clear difference between their optical signatures. We attribute this to the crosshatched fiber present in the material which is clearly visible in Figure 2. It is evident from the spectral measurements that this material absorbs light between $500-900 \mathrm{~nm}$, resulting in the qualitative difference between the materials' spectra. In plot $b$ of Figure 6, it can be seen that the reflectance of pristine Kapton falls below that of the aged materials at wavelengths smaller than $\sim 480 \mathrm{~nm}$. We attribute this to experimental artifact and/or a result of inaccurate background subtraction as the measurement at this wavelength is photon starved. The effect was not reproducible and is included only for completeness.

The absolute reflectance of Kapton decreases as electron dose increases. The change in reflectance is particular pronounced in the 500-900nm region. This region is particularly of interest as many of the telescopes currently in operation operate in the visible wavelengths $(\sim 300-1100 \mathrm{~nm})$. The degradation in optical reflectance is not homogeneous across this spectrum however, as is discussed below and shown clearly in Figure 7. This phenomenon and its application to space situational awareness will be discussed in the following section. 


\section{DISCUSSION}

The optical stability of the aluminum coated Mylar is physically reasonable as electron bombardment of metals can be expected to induce secondary electron emission, which is accounted for in the energetic dose calculations of the material, but should not affect its optical properties [25] and a temperature increase. While a sufficient temperature increase could lead to surface annealing, producing an overall smoother surface, the electron fluxes and material mounting methods used here are not sufficient to expect such dramatic temperature changes.[26] Each of the samples with Kapton on the surface underwent major changes in the absolute reflectance. This change in reflectance is due in part to the change in absorbance of the material. Since the Kapton is backed by a thin aluminum layer, the light that would otherwise pass through the Kapton is instead reflected back into the spectrometer. Pristine Kapton is fairly transparent between $500-700 \mathrm{~nm}$, the absorption edge at $500 \mathrm{~nm}$ is what gives Kapton its characteristic amber color. It has been shown in earlier studies that exposure to high energy, deeply penetrating electrons can cause extensive chemical damage to the material. The damaged material's new electronic structure results in resonant absorption of lower energy photons which results in a shift of the absorption band edge to around $730 \mathrm{~nm}$. This phenomenon manifests itself as a darkening of the material in the visible spectrum. [5, 11, 27]

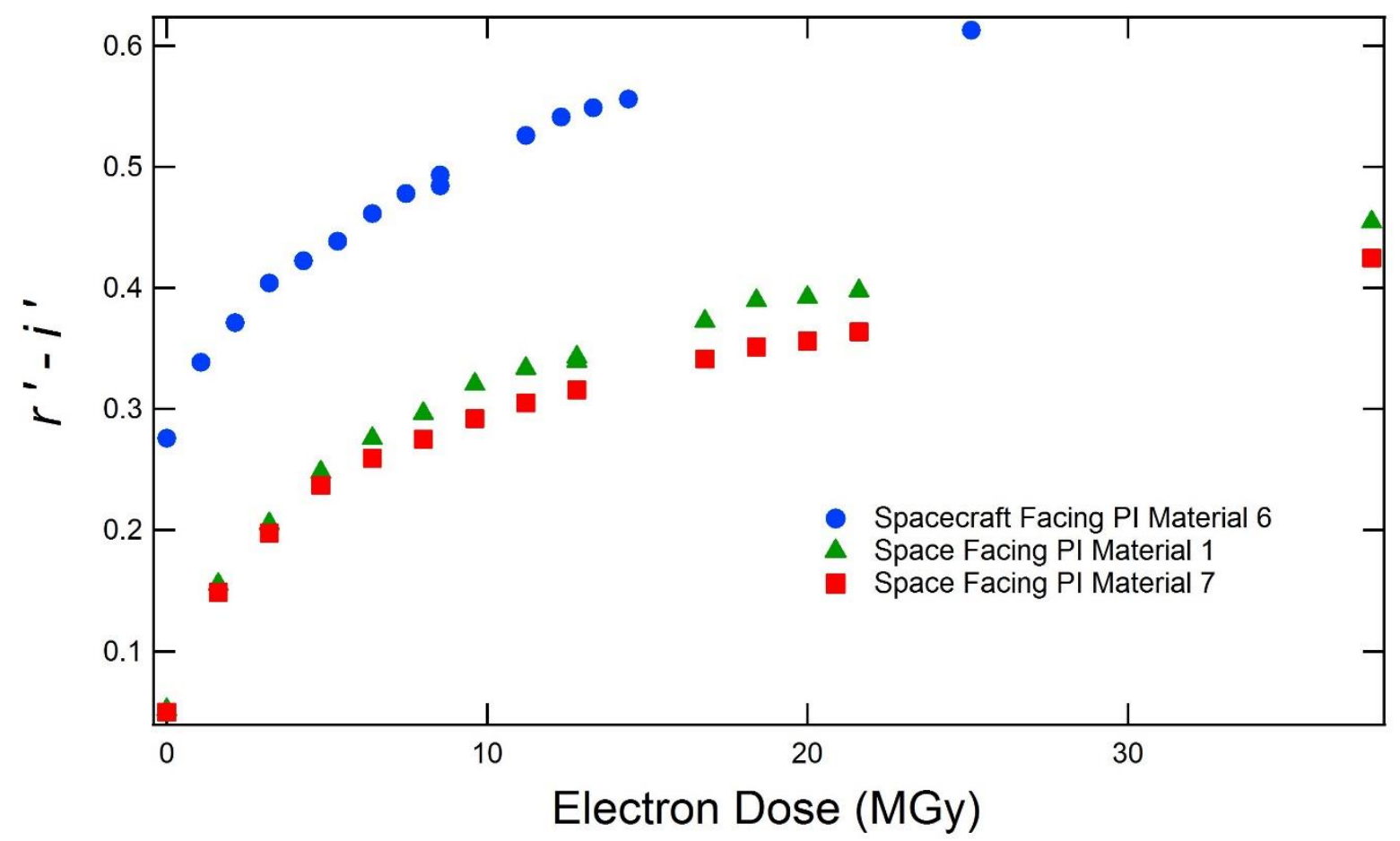

Figure 7. $r^{\prime}-i^{\prime}$ plots for materials 1,6, and 7. The magnitude of change for all materials is comparable. However, the absolute magnitude of the dose differs due to material composition. See text for further discussion.

The change in reflectance of the polyimide samples is as much as factor of 2 at some wavelengths. This has two effects on remote sensing. First, the material appears much less bright at the visible wavelengths and its apparent optical magnitude could be expected to drop significantly. Additionally, because the brightness of the material changes in a wavelength dependent fashion, color/color plots which can be generated using traditional astronomical filters will show distinct change as a function of a material's time on orbit. Color indices data is useful for identifying satellite features and general characterization of the target of interest, including signature classes (bus type or manufacture), feature extraction, and identifying how spectral/photometric responses over time due to space weathering. Using laboratory-based spectroscopic color indices can be used for comparative analysis with optical measurements acquired from telescopic observations in order to correlate remote and laboratory properties with the intent of ascertaining the intrinsic properties of the observed orbital debris or other targets of interest. Previous work used Johnson/Bessell filters to determine where specific materials fell in regard to the solar spectrum. Intact MLI (same sample as used for this study) resulted in a B-V $=+2.0$, B-R $=+2.9$, B-I $=+2.5$. [28] 
The MLI intact is constructed of multiple layers, with both outer faces composed of copper-colored Kapton (space Facing and spacecraft Facing), which has an absorption feature $480 \mathrm{~nm}$ (in the blue region and barely into the green region). The expected color index should show a higher signal return in the green, red, and infrared filters. The Sloan r' filter centered at $625 \mathrm{~nm}$ with a width of $140 \mathrm{~nm}$ and the Sloan i' centered at $770 \mathrm{~nm}$ with a width of 150 $n m .[16]$ As orbital debris is not emitting, but rather reflecting solar illumination response, $r^{\prime}-i^{\prime}$ for the Sun is +0.11 $( \pm 0.02)$.[29] Therefore, although the total absolute reflectance in the 400-1000 $\mathrm{nm}$ passband appears to decrease with higher -doses, the color-color spectral index range increases suggesting that for longer wavelengths the reflectance is increasing suggesting the electron dosing does contribute the reddening seen with Kapton materials. It should be noted that there are two data points on the color-color plot at the dose of $12.8 \mathrm{MGy}$ (sample 6) and 8.54 MGy (samples 1,7). The lower data points are for the same material measured after no additional electron bombardment, but 65 hours at room temperature in vacuum. This indicates that there is some degree of "material healing" in vacuum. While material healing in atmosphere has been well documented recently [5, 11, 27, 30], in order to create a model which gives us both predictive and characteristic power, the mechanisms of both damage and healing in a representative space-like environment must be thoroughly understood.

\section{CONCLUSIONS}

This study presents the optical effects of simulated aging experiments for different components of multilayer insulation. It has been shown that for Kapton, a ubiquitous spacecraft material, the changes that can be expected during an expected mission lifetime are of a magnitude to be useful when performing ground- and space-based optical characterization of materials on orbit.

\section{ACKNOWLEDGEMENTS}

We would like to acknowledge support from the Air Force Office of Scientific Research, Remote Sensing and Imaging Physics Portfolio (Dr. Stacy Williams). Grant 17RVCOR414.

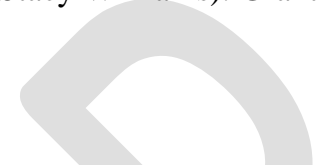

\section{REFERENCES}

1. K.J. Aberkromby, K.H., M. Guyote, J. Okada, E. Barker, , Remote and ground truth spectral measurement comparisons of FORMOSAT III, in Advance Maui Optical and Space Surveillance Technologies Conference. 2007: Maui, USA.

2. $\quad$ Bedard, M.D., Using a physics-based reflection model to study the reddening effect observed in spectrometric measurements of artificial space objects, in Advance Maui Optical and Space Surveillance Technologies Conference. 2007: Maui, USA.

3. $\mathrm{Wu}, \mathrm{Y}$., et al., A study on the free-radical evolution and its correlation with the optical degradation of $170 \mathrm{keV}$ proton-irradiated polyimide. Polymer Degradation and Stability, 2010. 95(7): p. 12191225.

4. Sun, Y.M., et al., The effects of high electronic energy loss on the chemical modification of polyimide. Nuclear Instruments \& Methods in Physics Research Section B-Beam Interactions with Materials and Atoms, 2002. 193: p. 214-220.

5. Daniel P. Engelhart, E.P., Sunita Humagain, Steven Greenbaum, Dale Ferguson, Russell Cooper, Ryan Hoffmann, Chemical and Electrical Dynamics of Polyimide Film Damaged by Electron Radiation. IEEE Transactions on Plasma Science, 2017. Accepted for publication(IEEE Transactions on Plasma Science Special Issue - Spacecraft Charging Technology - 2017).

6. Ginet, G., et al., AE9, AP9 and SPM: New models for specifying the trapped energetic particle and space plasma environment. Space science reviews, 2013. 179(1-4): p. 579-615.

7. Choi, H.S., et al., Analysis of GEO spacecraft anomalies: Space weather relationships. Space Weather-the International Journal of Research and Applications, 2011. 9.

8. Mehnert, R., Materials modification using electron beams, in Application of Particle and Laser Beams in Materials Technology, P. Misaelides, Editor. 1995. p. 557-580. 
9. Paulmier, T., et al. Electric properties of space used polymers under high energy electron irradiation. in Solid Dielectrics (ICSD), 2013 IEEE International Conference on. 2013. IEEE.

10. Meng, C., et al., Charging dynamics of a polymer due to electron irradiation: A simultaneous scattering-transport model and preliminary results. Chinese Physics B, 2012. 21(12): p. 127901.

11. D. Engelhart, E.P., D. Ferguson, R.Cooper, R. Hoffmann., Optical and Chemical Characterization of Polyimide in a GEO-like environment., in Advanced Maui Optical and Space Surveillance Technologies Conference. 2016: Maui, USA.

12. Plis, E., et al., Degradation of polyimide under exposure to $90 \mathrm{keV}$ electrons. physica status solidi (b), 2017.

13. M. Finckenor, D.D., Multilayer Insulation Material Guidelines, NASA, Editor. 1999, TP-1999209263.

14. H. M. Rodriguez, K.J.A., M. Mulrooney, E. Barker, Optical properties of multi-layered insulation, in Advanced Maui Optical and Space Surveillance Technologies Conference. 2016: Maui, USA.

15. Schildknecht, T., et al., Optical observations of space debris in GEO and in highly-eccentric orbits. Advances in Space Research, 2004. 34(5): p. 901-911.

16. Fukugita, M., et al., The Sloan digital sky survey photometric system. Astronomical Journal, 1996. 111(4): p. 1748-1756.

17. E. Plis, D.E., J. Likar, R. Hoffmann, R. Cooper, D. Ferguson, Electrical behavior of carbon-loaded Kapton for spacecraft applications. Journal of Spacecraft and Rockets, 2017. Submitted.

18. Mateo-Velez, J.C., et al., Severe Geostationary Environments: Numerical Estimation of Spacecraft Surface Charging from Flight Data. Journal of Spacecraft and Rockets, 2016. 53(2): p. 304-316.

19. Yue, L., et al., Effects of proton pre-irradiation on radiation induced conductivity of polyimide. Radiation Physics and Chemistry, 2016. 119: p. 130-135.

20. Khan, A.R., W. Jiang, and K. Toyoda, Electron and proton irradiation effect on bulk resistivity of polyimide measured in vacuum. 宇宙科学技術連合講演会講演集, 2013. 57: p. 6p.

21. Shi, J., et al., Optical properties and chemical structures of Kapton-H film after proton irradiation by immersion in a hydrogen plasma. Applied Surface Science, 2012. 258(8): p. 3829-3834.

22. Cooper, R. and R. Hoffmann, JUMBO SPACE ENVIRONMENT SIMULATION AND SPACECRAFT CHARGING CHAMBER CHARACTERIZATION. Air Force Technical Report, 2015. AFRL-RV-PS-TP-2015-0012.

23. Mosteller, R.D., Bibliography of MCNP Verification \& Validation: 1990-2003, in LA-UR-03-9032, L.A.N. Laboratory, Editor. 2003.

24. Coatings, A.A. http://www.acktar.com/category/BlackOpticalCoating. 2017.

25. Farnsworth, H.E., Electronic Bombardment of Metal Surfaces. Physical Review, 1925. 25(1): p. 41-57.

26. Experimental Innovations in Surface Science: A Guide to Practical Laboratory Methods and Instruments. 1997: Springer.

27. Plis, E., et al., Degradation of polyimide under exposure to $90 \mathrm{keV}$ electrons. Physica Status Solidi B-Basic Solid State Physics, 2017. 254(7).

28. Cowardin, H., P. Seitzer, K. Abercromby, E. Barker, and T. Schildknecht, Characterization of Orbital Debris Photometric Properties Derived from Laboratory-Based Measurements, in Advanced Maui Optical and Space Surveillance Technologies Conference. 2010: Maui, USA.

29. Sloan Digital Sky Survey. Available from: http://www.sdss.org/dr12/algorithms/ugrizvegasun/.

30. Humagain, S., et al., Study of Damage and Recovery of Electron Irradiated Polyimide using EPR and NMR Spectroscopy. Bulletin of the American Physical Society, 2017. 62. 